\title{
Extreme Limb Profiles of the Sun at Far-Infrared and Submillimeter Wavelengths
}

C. Lindsey, E.E. Becklin, F.Q. Orrall, M.W. Werner, J.T. Jefferies, and I. Gatley

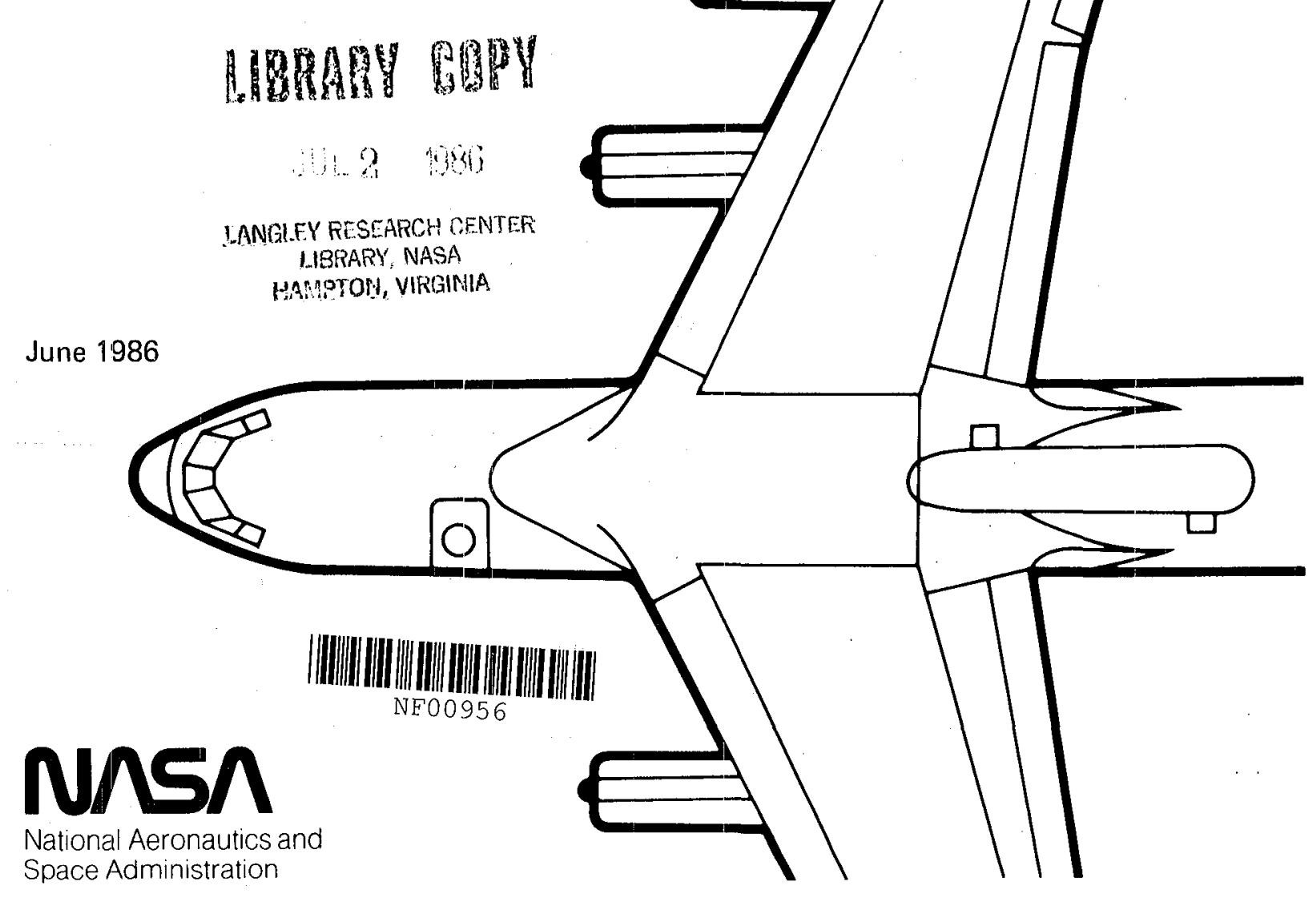




\section{Extreme Limb Profiles of the Sun at Far-Infrared and Submillimeter Wavelengths}

C. Lindsey,

E. E. Becklin,

F. O. Orrall, University of Hawaii, Institute for Astronomy, Honolulu, Hawaii

M. W. Werner, Ames Research Center, Moffett Field, California

J. T. Jefferies, National Optical Astronomy Observatories, Tucson, Arizona

I. Gatley, United Kingdom Infrared Telescope, Hilo, Hawaii

\section{NASA}

National Aeronautics and

Space Administration

Ames Research Center

Moffett Field, California 94035 


\begin{abstract}
We present $30,50,100$, and $200 \mu \mathrm{m}$ solar limb intensity profiles determined with arcsecond resolution from airborne observations of the occultation of the solar limb during the total eclipse of 1981 July 31. Two points of particular importance emerge: (1) The longerwavelength (100 and $200 \mu \mathrm{m})$ limbs are significantly brighter than disk center. At $200 \mu \mathrm{m}$ the extreme $1 \mathrm{imb}$ is about 1.22 times the brightness of disk center. This is consistent with the $6000 \mathrm{~K}$ temperatureplateau structure of the model chromospheres of Vernazza, Avrett, and Loeser (1973, Ap. J., 184, 605; 1981; Ap. J. Suppl., 45, 635) . The longer wavelength 1 imbs are extended significantly further above the visible limb than Vernazza, Avrett, and Loeser predict. These results provide a strong basis for modeling of the solar chromosphere free from the assumption of gravitational-hydrostatic equilibrium.

Subject headings: infrared: spectra - line profiles Sun: chromosphere - Sun: limb darkening - Sun: spectra
\end{abstract}




\section{INTRODUCTION}

In this paper we present results of analysis of far-infrared (30-200 $\mu \mathrm{m})$ airborne observations of the occultation of the solar limb during a total solar eclipse. The purpose of this analys is has been to determine the brightness profile of the extreme limb of the Sun at wavelengths in this range.

The far-infrared and submillimeter continuum from the sun originates in the lower and middle chromosphere. High-resolution limb brightness observations allow one to fix important constraints on both the temperature of the material and the density structure of the chromosphere. Infrared continuum radiation is important for studying the chromosphere because it emanates in local thermodynamic equilibrium (LTE) with the emitting material. Moreover, its source function is directly proportional to temperature by the Rayleigh-Jeans law. This makes it a uniquely valuable diagnostic for the temperature of chromospheric material.

The main obstacles to good far infrared observations are that (1) the Earth's atmosphere is opaque to the entire 50-200 $\mu \mathrm{m}$ continuum, and (2) far-infrared diffraction severely limits resolving power. The Kuiper Airborne Observatory (KAO) is uniquely suited to solve both of these problems, since it can fly a $0.9-\mathrm{m}$ telescope above $10 \mathrm{~km}$, where the atmospheric extinction is only a few percent (see Cameron, Bader, and Mobley 1971). On 1981 July 31 we used the KAO to observe the occultation of the solar limb during a total solar eclipse over the 
North Pacific. The observation was made simultaneously in well-defined bands at $30,50,100$, and $200 \mu \mathrm{m}$ and gave subarcsecond angular resolution (in the direction perpendicular to the limb).

For this paper we analyze the occultation observations already presented by Lindsey et al. (1983) to determine the limb brightness profile of the sun at $30,50,100$, and $200 \mu \mathrm{m}$. These profiles are compared with calculations of the submillimeter limbs of the model chromospheres of Vernazza, Avrett, and Loeser (1973, 1981; hereafter collectively VAL). These modern plane-parallel models rely on assumptions regarding gravitational-hydrostatic equilibrium to $\mathrm{f} i \mathrm{x}$ the vertical distribution of chromospheric material. We will see that the brightness of the extreme limb roughly agrees with that predicted by VAL at all wavelengths. However, the observed 100 and $200 \mu \mathrm{m} l \mathrm{jmbs}$ are extended significantly beyond the corresponding VAL limbs. This constitutes strong evidence for large departures from gravitationalhydrostatic equilibrium almost immediately above the chromospheric temperature minimum.

\section{DATA ANALYSIS}

\section{a) Data Acquisition}

The key feature of our observing technique was the use of the $\sim 100 "$ resolving beam of the KAO to isolate the point of contact on the lunar-solar crescent during the occultation. This provided a strong advantage in signal-to-noise ratio over earlier observations (Hagan 
1957; Hagan et al. 1971; Beckman, Lesurf, and Ross 1975; Clark and Boreiko 1982) in integrated Sun-Moon radiation. The detector system we used observed the same $100 "$ field of view simultaneously at all four wavelengths. The passbands of the infrared filters used are described in Table 1.

In ideal circumstances the observation could be done quite simply by centering the telescope beam on the solar crescent at the point of contact and observing the integrated radiation in the 100 " telescope beam. Reality, however, presents two main complications: (1) infrared emission from the sky and telescope produce a large varying signal that must be corrected, and (2) the KAO is subject to tracking errors, which can introduce artificial intensity variations as well. To solve these problems, we introduced a special two-beam scanning scheme, illustrated in Figure 1.

The first problem was solved by the standard method of two-beam chopping. In this scheme the telescope observes in two separate beams separated by $2^{\prime}$ on the sky (Fig. 1a); the signals in these beams are electronically subtracted, canceling telescope and sky emission.

The optical and electronic schemes for doing this are described briefly in Lindsey et al. (1983). We define the "positive beam" and the "negative beam" according to the sign of the signal voltage produced by an infrared source in the respective beam.

The second problem was solved by scanning this two-beam configuration repeatedly across the solar crescent in a 
triangular-wave fashion (Fig. 1b), assuring that the crescent image passed through both bean maxima once each second. The resulting signal profile is illustrated in Fig. 1c. As the negative beam sweeps across the limb crescent, a negative signal (two-beam difference) results. This is closely followed by a positive deflection as the positive beam follows behind. The amplitude of this sigmoid profile serves as a measure of the integrated solar intensity in the band swept by the beams. The quantitative parameters relating to the two-beam chopping-scanning scheme we used are summarized in Table 2.

\section{b) Description of Occultation Data}

Figure 2 shows how the profile in Figure 1c evolves during occultation of the solar limb. Here the actual $100 \mu \mathrm{m}$ signal during the limb occultation at second contact is shown. Profile a shows the triangular-wave scan profile of the point half way between the two beams during the scan. The peak-to-peak amplitude of the scan position, $x$, is 5 arcmin, with positive $x$ denoting outward from the limb. During each linear scan segment, the $100 \mu \mathrm{m}$ difference signal, $\mathrm{V}$ (profile $\mathrm{b}$ ), undergoes a positive excursion and a negative one as the positive and negative beams pass in turn over the solar crescent. These two-bean difference excursions diminish as the solar crescent disappears.

The rate of motion of the lunar limb with respect to the Sun was $0 . " 459 s^{-1}\left( \pm 0 . " 001 s^{-1}\right)$. So in each scan a $0 . " 459$ segment of the solar limb was occulted. This represents our sampling-limited angular resolution perpendicular to the limb (see Table 2). 
The infrared limb profiles are fixed with respect to the position of the visible limb by timing the infrared observations against the disappearance of the visible solar limb. In Figure 2, we see at the disappearance of the visible limb $(t=0)$ that considerable solar infrared emission persists. Note also that after the Sun has been completely occulted (e.g., $t>10 \mathrm{~s})$, lunar emission remains clearly visible.

\section{c) Reconstruction of the Solar Limb Profile}

The crux of the task of reconstructing the solar limb profile is to take a proper account of the two-beam response profile of the telescope in the observation. The procedure for this is summarized in this section. A more detailed discussion of some of the important concepts is presented in Appendices A and B.

The signal profiles of Figure $2 b$ are a convolution of the twodimensional beam-response profile of the telescope with the twodimensional lunar-solar intensity distribution. This two-dimensional convolution can be simplified and reduced to a one-dimensional convolution along the direction of scan of one-dimensional counterparts of the telescope response profile and the solar intensity profile (Appendix A). In this formalism, the effective onedimensional profile of a uniform intensity terminated by the lunar limb is simply a step function, while the one-dimensional profile for a thin crescent much narrower than the resolving beam approaches a Dirac delta function. 
The reconstruction of the solar limb brightness profile is done in time reverse order of the occultation. Starting well into totality $(t>15 \mathrm{~s})$, the lunar emission is modeled as a step-function brightness profile convolved with the known two-beam response profile of the telescope. The solar intensity is reconstructed inward from the limb (regressively in time). For each scan across the solar crescent, we assume that the intensity of the current solar segment over which the lunar limb moves during that scan is constant. (It actually varies, of course, but we do not attempt to resolve variations finer than the sampling interval.) This "average intensity over the scan" is fixed so that its contribution, along with the outward lying profile (already determined), fits the observations: that is, we numerically convolve our occulted model limb profile with the telescope beam, treating the intensity in the current segment as a free parameter adjustable to optimize agreement with the observations in the current scan. The result for the $100 \mu \mathrm{m}$ data shown in Figure $2 b$ is the limb intensity profile plotted in Figure $2 c$.

\section{d) Calibration}

To relate the brightness of the solar limb to the brightness at disk center, the telescope response profile must be calibrated near disk center. We accomplished this by scanning the lunar-solar discontinuity across the anticipated point of second contact on the lunar limb earlier, while it passed over quiet Sun near disk center 20 minutes before totality. This configuration provided a "step-function discontinuity" of known magnitude with which to calibrate the 
telescope response profile for analysis of the limb observation that followed. The application of this calibration to determine the limb brightness in terms of the disk-center brightness also is greatly simplified by reduction to a single dimension. wherein the scan profile across the lunar-solar discontinuity is represented by the one-dimensional telescope response profile convolved with a one-dimensional step function (Appendix A).

This calibration is, of course, crucial in setting all of the ordinate scales of the limb profiles plotted in this paper. We note on this basis, for instance, that the $100 \mu \mathrm{m}$ limb profile (Fig. 2c) culminates to an intensity approximately $15 \%$ brighter than at disk center before dropping rapidly to zero several arcseconds above the visible limb.

\section{e) Some Useful Considerations}

Note that when the solar crescent is much narrower than the telescope resolving beam $\left(\sim 100^{\prime \prime}\right)$, the limb intensity is approximately the derivative of the occultation curve. However, as the solar crescent approaches the resolving beamwidth, the occultation curve begins to saturate. An accurate measurement of the beam profile calibrated to the disk-center intensity of the Sun allows a straight-forward solution by the above method for the limb intensity profile extending inward approximately a beamwidth (100") from the extreme limb. Taking this approach, we find that the $15 \%$ limb brightness excess seen at $100 \mu \mathrm{m}$ develops relatively smoothly starting about 30" inside the solar limb. 
Let us now examine the lunar contribution noted in Figure $2 \mathrm{~b}$ : Comparing the lunar signature during totality with the calibration scans, we Pind the $100 \mu \mathrm{m}$ lunar intensity to be 0.011 times the intensity of the Sun at disk center. Taking the brightness temperature of the Sun at disk center to be $4350 \mathrm{~K}$ (VAL), we find the temperature of the Moon (dark side most recently illuminated by the sun) to be $100 \mathrm{~K}$ (emissivity assumed unity). (Note that for this temperature, $100 \mu \mathrm{m}$ is no longer in the Rayleigh-Jeans region.) This is consistent with Linsky (1973).

If one simply looks at the envelope of the scan profiles of Figure $2 b$, it is seems that the solar contribution disappears by $t \sim 4 s$, well before the reconstructed limb profile drops to zero. In fact a significant solar contribution is still present, but is hidden by the lunar residual. A small solar contribution manifests itself as an apparent outward motion of the lunar limb rather than in adding to the amplitude of the lunar profile. A determination of the limb profile in this region rests on accurate tracking of the telescope on the lunar limb. These considerations are explained and clarified in Appendix B.

Because it is so important that we know the brightness scale of the limb profile in terms of the disk-center brightness of the Sun, it is useful to have an additional comparison to provide an independent calibration. To accomplish this, we scanned the solar limb at the anticipated point of second contact immediately before 
and after the calibration scans (across the lunar limb near Sun center) were made. The limb profiles reconstructed as described in $\S \S$ IIC and IId were then artiflcially scanned by numerically convolving them with the telescope response profile, and the results were compared with the observed limb scans. The artificial scans agreed with the observations to within $4 \%$ or better (depending on wavelength). This small discrepancy was attributed to drift in instrumental sensitivity (or possibly even atmospheric transparency) during the 20 minutes between the calibration observations and the occultation observation. The limb profiles reconstructed by the method described in $\S$ IIC and IId have thus been renormalized to correct for this small drift.

While we have illustrated our data analysis techniques using only the $100 \mu \mathrm{m}$ data as an example, the same procedure has been applied independently and identically to all four wavelengths. No assumptions have been made about the brightness profile for any wavelength to determine that of any other.

\section{I . RESULTS}

Some of the circumstances of the 1981 eclipse as we observed it are summarized in Table 3 . As described in Lindsey et al. (1983), we observed both the second-contact occultation of the solar limb and the reappearance of the third-contact limb following totality. The third-contact region was contaminated with weak 
calcium plage (Hale regions 17754 and 17761). The quality of these data was also somewhat compromised by tracking instabilities that developed as the limb reappeared (see Appendix B). We first concentrate on the quiet second-contact limb.

\section{a) Second-Contact Limb}

Figure 3 shows the intensity profiles determined for the second-contact limbs at $200,100,50$, and $30 \mu \mathrm{m}$ (solid curves a, b, c, and $d$, respectively). In all four profiles we see the observed brightness sustained near unity or above up to or past the visible limb before dropping to zero over an interval of about 2 ". Convolved with the solar limb profile is the height distribution of the lunar limb, whose width is about $0 . " 5$ in the resolving beam at the second contact limb. We make no attempt to deconvolve this narrow roughness distribution in this paper.

Also shown in Figure 3 are the corresponding limb-intensity profiles of the VAL (1973) chromosphere, computed by Hermans (1984). These profiles are identical (within observational errors) to those resulting from Model $C$ of VAL (1981). All profiles in Figure 3 are normalized to unity brightness at disk center (left ordinate scale) and to the corresponding VAL disk-center brightness temperature (right ordinate scales). (Note that we ourselves do not in any way measure the absolute brightness temperature of the Sun, but rather we compare the limb brightness with the disk-center brightness.) In this figure, the visible limb (denoted by vertical arrows) is taken to be at a 
height of $340 \mathrm{~km}$ above the base of the photosphere, in accordance with VAL .

The error bars shown for heights greater than $-1000 \mathrm{~km}$ in Figure 3 denote a statistical uncertainty in average intensity of one standard deviation over a $1 "$ segment of the limb profile. That is, for the errors shown here, one assumes an effective resolution of $1 "$. These errors are based on noise in the reconstructed profile appearing above $3200 \mathrm{~km}$ in Figure 3 , at which point we assume that the sun is no longer contributing radiation.

For lower heights, below about $-1000 \mathrm{~km}$, photometric errors can cause significant artificial variations in the slope of the occultation curve, and the uncertainties are, thus, increased. The relative depressions in intensity in the height interval from -3200 to $-900 \mathrm{~km}$ could, for instance, be caused by a $2-4 \%$ decrease in transmission lasting $4 \mathrm{~s}$. Variations such as this, small not only in optical density but in temporal extent, become increasingly important for a wider solar crescent, hence greater uncertainties. The error bars shown for heights $<-1000 \mathrm{~km}$ in $\mathrm{Figure} 3$ correspond to the error that would be introduced by a $5 \%$ change in atmospheric transmission lasting for $4 \mathrm{~s}$. Our repeated calibrations scans made $\sim 20 \mathrm{~m}$ before second contact show a stability within this limit for all four wavelengths.

A close examination of Figure 3 suggests that the 100 and $200 \mu \mathrm{m}$ limbs maintain an overall intensity significantly greater than disk center for heights from $-3000 \mathrm{~km}$ up to the base of the chromosphere at 
$350 \mathrm{~km}$. This is in agreement with the limb profiles computed for the VAL. These limb excesses are found to develop relatively smoothly starting within $40^{\prime \prime}$ inside the visible limb. (This will be characterized more formally in a forthcoming publication.)

In Table 4 we show the limb intensities observed at second contact and computed for the VAL averaged over the height interval from $-3000 \mathrm{~km}$ to $+350 \mathrm{~km}$ for all four wavelengths. We see 100 and $200 \mu \mathrm{m}$ limb excesses ranging from $12 \%$ to $22 \%$, roughly consistent with or slightly greater than the VAL intensities. For 30 and $50 \mu \mathrm{m}$ the limb intensity averaged over the above interval is approximately equal to or slightly greater than the disk center intensity. However, these observed 30 and $50 \mu \mathrm{m}$ intensities are significantly greater than the VAL intensities, which show an extreme limb up to $10 \%$ darker than disk center.

Note that the errors assigned to the limb intensities averaged over the 3000 to $+350 \mathrm{~km}$ interval are considerably smaller than those shown by the error bars in Figure 3 . It is important to realize that the errors corresponding to a broader height range are much less than for the constituent narrower height intervals. This is only partly because of increased statistics in the greater time interval. The limb intensity can be thought of as the derivative of an occultation curve. And over a larger time interval, a proportionately greater signal difference presents itself. Alternatively, a greater time base over which to measure the derivative results in a more accurate 
determination even without increased statistics. The uncertainty expected due to random noise in an occultation measurement is, thus, proportional to the time resolution to the power of $-3 / 2$, rather than to the power of $-1 / 2$ as it is for simple photometry.

Finally, the observed $30 \mu \mathrm{m}$ limb is seen to coincide reasonably in height with the $30 \mu \mathrm{m}$ limb of VAL. A clearly different situation develops at the longer wavelengths: the observed 100 and $200 \mu \mathrm{m}$ limbs are extended outward considerably further than the VAL 1 imbs. Significant $200 \mu \mathrm{m}$ emission is detected nearly $1,000 \mathrm{~km}$ above the top of the VAL 1 imb cutoffs. This important result is consistent with the analysis of Lindsey et al. (1983). They found somewhat greater limb extensions in their preliminary analysis of the occultation curves, because they neglected the effect of increased brightening toward the limb at longer wavelengths.

\section{b) Third-Contact Limb}

The limb-brightness profiles reconstructed from the third-contact observations are shown in Figure 4 . The 30 and $50 \mu \mathrm{m}$ profiles are similar to those of the quiet second-contact limb. The 100 and $200 \mu \mathrm{m}$ profiles are somewhat brighter and more extended in height above the visible limb than their second-contact counterparts.

Because of considerably larger uncertainties in telescope tracking, both along the direction of scan (where lunar motion generates an artificial solar contribution for $\mathrm{h}>1000 \mathrm{~km}$ ) and perpendicular to it (where plage can contribute an artificial 
variation), the uncertainties in the reconstructed profile are much greater (see Appendix B). The large intensity spike at $\mathrm{h}=2000 \mathrm{~km}$ is not significant, nor is the large dip at $1000 \mathrm{~km}$.

Their cumulative contribution is, however, significant. The solar occultation curve determined for $\mathrm{h}<1000 \mathrm{~km}$ (where motion across the lunar limb cannot be confused with a solar contribution) extrapolates to zero at about $h=2400 \mathrm{~km}$, far above the VAL limb (Lindsey et al. 1983). Telescope tracking errors can obscure the detajled distribution of the extended limb material in the time interval in which a solar contribution can be confused with tracking errors on the lunar limb. However, following this time interval, when the solar contribution becomes obvious, the occultation curve represents the solar intensity integrated from thence outward. That this integrated limb excess is greater for the longer wavelengths at the third contact limb than at the second contact limb is highly significant and is not a manifestation of tracking errors.

\section{DISCUSSION AND SUMMARY}

The use of narrow-beam observations at the point of contact in a total eclipse to determine the solar limb brightness profile has so far been unprecedented in the infrared and submillimeter continuum. It should be clear that this technique is very powerful and can give results of far higher quality at the extreme limb than full-disk observations. The limb profiles we have obtained can serve as a 
valuable tool for modeling of the solar chromosphere independent of assumptions regarding gravitational-hydrostatic equilibrium.

The results of our analysis seem to be consistent with the full-disk observations of Clark and Boreiko (1982). Moreover, our results are also consistent with those of Lindsey et al. (1984), who observed gradual darkening approaching the limb at $30,50,100$, and $200 \mu \mathrm{m}$. As they anticipated, this darkening is reversed at the extreme limb. The 100 and $200 \mu \mathrm{m}$ limb excesses shown by our analys is are two

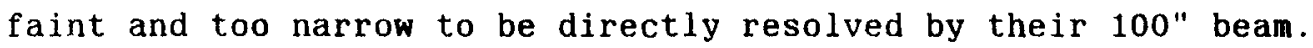

The agreement in our 100 and $200 \mu \mathrm{m}$ limb brightness observations with the VAL supports the temperature structure of VAL approaching the $6000 \mathrm{~K}$ plateau. The infrared continuum opacity is essentially entirely $\mathrm{H}^{-}$free-free and $\mathrm{H}^{0}$ free-free absorption, so the infrared continuum is emitted in LTE with the electron temperature. Material observed inside the $200 \mu \mathrm{m}$ limb must therefore be about $6000 \mathrm{~K}$ or cooler, in keeping with VAL. Even in an inhomogeneous chromosphere, a substantial fraction of much hotter material would raise the limb brightness considerably, since hydrogen suddenly becomes highly ionized (and therefore opaque) above $6000 \mathrm{~K}$. However, if different regions at the same height can have different temperatures, then a significant fraction of the chromospheric medium could be considerably cooler than $6000 \mathrm{~K}$. Because opacity becomes such a strong function of temperature at $6000 \mathrm{~K}$, cool material could be transparent to $200 \mu \mathrm{m}$ radiation even at a relatively high density and thus go unseen. 
A more careful analysis with more modern atmospheres should be useful in considering the $30 \mu \mathrm{m}$ brightness discrepancy between the observed limb and VAL. More recent work (e.g., that summarized by Avrett 1985) argues for a hotter temperature minimum. This may alleviate the discrepancy at $30 \mu \mathrm{m}$ without perturbing the agreement at the longer wavelengths.

Earlier models of spicules (see Beckers 1972) propose relatively high temperatures $(9000-16,000 \mathrm{~K})$ over a large range in height. Our results appear to rule out such high temperatures at elevations much below $2000 \mathrm{~km}$. We anticipate that our observations will require denser material up to this elevation than earlier models predict. Recent work by Landman (1984) also seems to favor cooler chromospheric material at lower elevations.

The appearance of extended limb emission remains one of the most important results of the KAO eclipse observation. The 100 and $200 \mu \mathrm{m}$ limb extensions we have observed are clearly greater than those predicted by VAL. This indicates the existence of relatively dense $\operatorname{cool}(\leq 6000 \mathrm{~K})$ material well above the heights predicted by the VAL, based on assumptions regarding gravitational-hydrostatic equilbrium. A smaller limb extension is apparent even in the $50 \mu \mathrm{m}$ profile. Extended limb material at such short wavelengths strongly suggests a coincidence between the onset of significant chromospheric heating and a marked departure of the chromospheric medium from gravitational-hydrostatic equilibrium. 
In sunmary, we emphasize two important points: (1) The infrared brightness profiles we have determined are consistent with the temperature structure of VAL, particularly in the sense that the infrared limb is formed by relatively dense cool material with a temperature up to but not far exceeding $6000 \mathrm{~K}$. However, (2) the observations show a need for correcting the assumption of gravitational-hydrostatic equilibrium in the middle chromosphere as low as $1000 \mathrm{~km}$. Observations such as these can serve as a strong basis for such a correction. We are presently modeling smooth perturbations of the VAL from gravitational-hydrostatic equilibrium to fit our observations. In principle, accurate infrared limb observations of a smooth atmosphere over a reasonable range in wavelength are sufficient for modeling the opacity and temperature of the atmosphere to within the resolution of the observations. This generality can even be extended to a rough atmosphere, such as one composed of spicules, if the density and morphology of the spicules are independently known.

\section{ACKNOWLEDGMENTS}

We wish to thank Mr. Kevin Krisciunas for his special efforts in support of this work. Particularly in view of the difficult and special demands of these observations, his hard work and excellent performance in this project were of tremendous value. We thank Miss Linda Hermans and D. Braun for calculating the limb profiles of the VAL atmosphere and Dr. J. Heasley for assistance in these calculations. This work was supported by NASA grants NAGW 723 and NGL 12-001-011. 


\section{APPENDIX A \\ RECONSTRUCTION OF THE LIMB BRIGHTNESS PROFILE}

The problem of accounting for the effect of a relatively broad and irregular telescope beam in a limb occultation observation is greatly simplified by eliminating from consideration smearing of the image along the lunar $1 \mathrm{imb}$, along which we do not attempt to obtain enhanced resolution. The purpose of the following line of reasoning will be to do this, reducing the problem of smearing of an image by a two-dimensional telescope beam to a one-dimensional convolution.

Let us denote the intensity of the observed source by a spatial source function $s(r)$, where $r$ is a two-dimensional displacement vector denoting a point $P(r)$ on the sky referenced to the center, 0 , of the telescope beam profile (see Figure A1). The signal response of the telescope takes the form

$$
V=\int d^{2} \mathbf{r} b(\mathbf{r}) \mathbf{s}(\mathbf{r})
$$

where $b(r)$ is the two-beam difference signal of the telescope in response to a unit point source at $\mathbf{r}$.

We now introduce the trajectory, L, along which we intend to scan the bean. Here we refer the reader to Figure $A 1$. Let $e$ be the unit vector parallel to $L$ pointing outward from the solar limb. Now let $\mathbf{x}=\mathbf{r} \cdot \mathbf{e}$ be the displacement of a point, $P(\mathbf{r})$, along the scan trajectory. (Note that $L$ is not quite perpendicular to the limb at the point of contact. In our observation, L was parallel to the local horizon, and was incident to the limb at $20^{\circ}$ at the point of contact; see Table 3.)

We now proceed to express the integral A1 in a special way, that 
is, in terms of line integrals along certain curves, $\Gamma$, belonging to the family of loci with which the lunar limb will coincide at various times throughout the occultation. We will identify a particular curve in this family by the notation $\Gamma(x)$," where $x$ is the displacement along $L$ of the point where the curve $\Gamma$ intersects $L$. Then we can express $V$ as

$$
v=\int d x f_{\Gamma(x)} d y b(r) s(r)
$$

were $d y=|d r \times e|$ is the differential progression of $\Gamma$ perpendicular to the $x$ direction, and the integral in the $x$-direction is simply a conventional integral along $L$. (Let us caution the reader not to mistake the line integral along $\Gamma$ for a simple integral in the $y$-direction.)

At this point, we introduce the assumption that the solar intensity is dependent only on radial distance from the Sun's center. While the above assumption may not be accurate, we must make it as a concession that we cannot attempt to use the lunar limb to resolve structural dependence parallel to it. The radial distance from sun center is, in turn, constant along $\Gamma$ to within $0 . " 1$ in the resolving beam of the telescope. We will now add the simplifying assumption that the lunar limb moves in the $x$-direction without changing shape or orientation. This allows us to say that if

$$
P(\mathbf{r}) \in \Gamma(x)
$$

then

$$
P(\mathbf{r}-\mathbf{e} \xi) \in \Gamma(\mathbf{x}-\xi)
$$


That is, if $r$ identifies a point on the curve $\Gamma(x)$, then $r-e \xi$ specifies a corresponding point on $\Gamma(x-\xi)$. (For a circular arc, this is exactly true only if the limb moves parallel to the direction of scan, which it does not. However, for us it is true to within $0 . " 1$, even though actual features on the lunar limb move $20: 4$ from the $x$-direction (see Table 2).

Since $s$ is constant along $\Gamma$, we can remove it from the integral along $\Gamma$. Then $V$ takes the form

$$
V=\int d x S(x) B(x)
$$

where

$$
B(x)=f_{\Gamma(x)} d y b(r),
$$

and $S(x)$ is simply $s(r)$ for $P(r) \in \Gamma(x)$. We can think of $B(x)$ as the effective one-dimensional point-source response of the telescope. When the two dimensional function, $s(\mathbf{r})$, takes the form of an intense narrow crescent conformal with the lunar limb, the effective onedimensional source function becomes a Dirac delta function, $\delta(x)$, whereby equation (A4) then equates $V$ with $B$. Now if we translate the beam configuration across the source in the $x$-direction a distance $\xi$, equation (A2) becomes

$$
V(\xi)=\int d x f_{\Gamma(x)} d y b(r-e \xi) s(r)
$$

Extracting $s$ as before and then applying equation (A3), we see that

$$
\begin{aligned}
V(\xi) & =\int d x s(x) \int_{\Gamma(x-\xi)} d y b(r) \\
& =\int d x s(x) B(x-\xi)
\end{aligned}
$$


This reduces the problem of describing the effect of the resolving beam to a simple one-dimensional convolution, once we have determined B. Fortunately, B can be measured directly by scanning the lunar limb as it passes over the quiet sun near disk center, bypassing the unpleasant task of somehow measuring $b(r)$ and computing the line integrals in equation (A5). The one-dimensional source function for a uniform background of unit (quiet-solar) intensity occulted by a perfectly dark lunar limb is simply a step function, $\theta(x)$. The calibration profile $C(x)$ that results from scanning the lunar limb at the predetermined point of second contact is simply the convolution of $\theta(x)$ with the telescope beam. Since the Dirac delta function, $\delta(x)$, is simply the derivative of $\theta(x)$, the one-dimensional point-source of the telescope, $B(x)$, is simply the derivative of the calibration profile:

$$
B(x)=\frac{d}{d x} c(x)
$$

During totality, we scan the lunar limb by itself to obtain a correction for its small perturbation to the unit solar step function assumed in the earlier calibration scan. The resulting profile (see Fig. 2b) is identical in shape to the profile of the occulted quiet Sun near disk center but, naturally, of much smaller ampiitude. We therefore represent the Moon by a simple step function as well.

The effect of the lunar limb on the solar limb profile can now be expressed by simply truncating the solar source profile, that is, multiplying it by a step function whose cutoff progresses outward as the occultation proceeds. Modeling this process is best done in 
time-reverse, looking first at totality, modeling the lunar contribution, and proceeding backwards as solar contributions now appear. The data set is divided into $1 \mathrm{~s}$ time intervals coincident with individual scans across the solar crescent. In each such time interval, a $0 . " 469$ segment: of the solar limb is uncovered. The intensity in each such segment is assumed constant. For a given intensity we can model the process of this segment being uncovered as the lunar limb "recedes." Its contribution is added to the outer-lying profile (already determined), the entire profile up to the lunar limb being convolved with the telescope beam, $B$. The intensity in the segment being uncovered is selected so that this convolution fits the observed profile for the scan under consideration.

It should be clear that near the extreme limb, the brightness profile is proportional to the derivative of the occultation curve (the envelope of the scans in Fig. 2b). Inward from the limb this relationship fails as the solar crescent saturates the resolving beam. The procedure described above compensates for this saturation and can recover the $1 \mathrm{imb}$ profile as far back as $90^{\prime \prime}$. We find for all wavelengths that the brightness profile glides smoothly from its limb value to disk center value within about $40^{\prime \prime}$ inside the extreme limb. 
APPENDIX B

APPARENT MOTION OF THE LUNAR LIMB

It is important to notice, when examining Figure 1 , that the envelopes of the lunar residual $(t>10 \mathrm{~s}$ ) and the solar crescent without the Moon do not simply add. Since they have different signatures, the local maximum of their sum is not equal to the sum of their maxima. In fact, in the time interval $4 \mathrm{~s}<\mathrm{t}<7 \mathrm{~s}$, when the solar contribution is small compared to the lunar, the effect of the solar contribution is indistinguishable from a motion of the lunar limb outward with no discernible change in amplitude. This is shown in the following argument.

Following Appendix A, we represent the one-dimensional lunar brightness profile by a step function:

$$
S_{m}(x)=I_{m} \theta(x)
$$

The one-dimensional brightness profile of a narrow solar crescent can be represented by a delta function of integrated brightness $I_{0} h$ :

$$
S_{S}(x)=I_{0} h \delta(x) \text {, }
$$

where $I_{0}$ is the average intensity of the solar crescent, and $h$ is its width. Differentiating equation (B1), noting that the derivative of $\theta(x)$ is $\delta(x)$, and comparing with equation (B2), we find that

$$
S_{s}(x)=\frac{I_{o}}{I_{m}} h \frac{d}{d x} S_{m}(x) .
$$

This relation is preserved under convolution with the telescope beam profile: 


$$
v_{s(x)}=\frac{I_{0}}{I_{m}} h \frac{d}{d x} v_{m}(x) .
$$

The sum of $v_{m}$ and $v_{s}$,

$$
V(x)=V_{m}(x)+\frac{I_{0}}{I_{m}} h \frac{d}{d x} V_{m}(x)
$$

is simply the first-order Taylor series expansion of

$$
V_{m}\left(x+\frac{I_{0}}{I_{m}} h\right) \text {. }
$$

Thus for $4 \mathrm{~s}<t<7 \mathrm{~s}$, the persistence of solar radiation manifests itself by an apparent outward motion of the lunar signature a distance

$$
\Delta \mathrm{x}=\frac{\mathrm{I}_{\mathrm{O}}}{\mathrm{I}_{\mathrm{m}}} \mathrm{h} .
$$

For example, for a solar crescent of disk-center intensity, $I_{0} / I_{m} \sim 90$. Thus, the apparent outward motion of the lunar limb would be 90 times the width of the solar crescent.

A careful comparison of the scan-pairs labeled p, q (at $t=5.5 \mathrm{~s}$ ) and $r, s$ (at $t=9.5 \mathrm{~s}$ ) in Figure $2 \mathrm{~b}$, for instance, shows a 15" outward displacement of the former relative to the latter. This displacement is equivalent to a solar contribution from a $0 . " 17$ crescent of unit (disk-center) intensity (or, for instance, a 1."7 crescent of intensity 0.1 ). Estimates of the real tracking uncertainty based on (1) scans of the Moon during totality, (2) scans of the solar crescent approaching totality, and (3) a study of a video reference monitor approaching visible second contact indicate a 
maximu tracking uncertainty of order 4" during the second-contact observation. On this basis, we can conclude that the $100 \mu \mathrm{m}$ solar residual seen up to $t=8 \mathrm{~s}$ is primarily real and statistically significant, and not, for instance, a manifestation of lunar tracking errors.

The same cannot be said for the third contact observation $88 \mathrm{~s}$ later. Partly because of difficulties in setting up a pointingscanning configuration on the opposite lunar limb without visual reference to a bright solar crescent, tracking instabilities of order 10" appeared during third contact, introducing errors that limit our discrimination of the third-contact limb. 
Table 1

Filter Passbands

\begin{tabular}{lcc}
\hline & $\begin{array}{c}\text { Mean wavelength } \\
(\mu \mathrm{m})\end{array}$ & $\begin{array}{c}\text { Bandwidth } \\
(\mu \mathrm{m})\end{array}$ \\
\hline a & 200 & 60 \\
b & 100 & 30 \\
c & 50 & 15 \\
d & 30 & 9 \\
\hline
\end{tabular}


Table 2

Observing Parameters

\begin{tabular}{lc} 
Beam separation & $2^{\prime}$ \\
Length of scan & $5^{\prime}$ \\
Resolution (beam width) & $\sim 1.5^{\prime}$ \\
Sampling interval & $1 \mathrm{~s}(0 . " 469)$ \\
\hline
\end{tabular}


Table 3

Eclipse Circumstances

\begin{tabular}{|c|c|c|}
\hline & $\begin{array}{l}\text { Second } \\
\text { Contact }\end{array}$ & $\begin{array}{l}\text { Third } \\
\text { Contact }\end{array}$ \\
\hline Time (UT) & $0424 \quad 16.5$ & 042544.2 \\
\hline \multicolumn{3}{|l|}{$\begin{array}{l}\text { Position of Contact } \\
\text { Points: a }\end{array}$} \\
\hline $\begin{array}{l}\text { Longitude } \\
\text { (Carrington } \\
\text { Rotation \#1711). }\end{array}$ & $156^{\circ}$ & $336^{\circ}$ \\
\hline Latitude (degrees) & $12^{\circ} \mathrm{S}$ & $12^{\circ} \mathrm{N}$ \\
\hline $\begin{array}{l}\text { Angle of contact point } \\
\text { (degrees above local } \\
\text { horizontal) }\end{array}$ & $20.4^{\circ}$ & $-20.4^{\circ}$ \\
\hline $\begin{array}{l}\text { Occultation Speed: a } \\
\text { (Lunar Limb relative } \\
\text { to Solar Limb, } \\
\text { arcsec } s^{-1} \text { ) }\end{array}$ & 0.469 & 0.469 \\
\hline Limb Activity: & Quiet & $\begin{array}{l}\text { Weak (Dying) } \\
\text { Plage (Hale } \\
\text { Regions } 17754 \\
\text { and 17761) }\end{array}$ \\
\hline
\end{tabular}

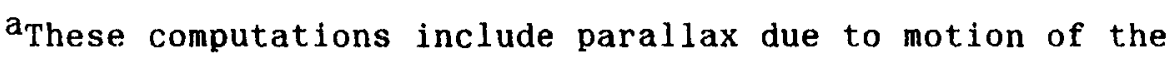
aircraft. 
Table 4

Limb Brightnesses ${ }^{a}$

\begin{tabular}{|c|c|c|}
\hline \multirow{2}{*}{$\stackrel{\lambda}{(\mu \mathrm{m})}$} & \multicolumn{2}{|c|}{$\begin{array}{c}\left\langle\mathrm{I} / \mathrm{I}_{\mathrm{O}}\right\rangle \\
(-300 \text { to }+350 \mathrm{~km})\end{array}$} \\
\hline & Observed & VAL \\
\hline 30 & $1.02 \pm 0.05$ & 0.91 \\
\hline 50 & $1.07 \pm 0.05$ & 0.95 \\
\hline 100 & $1.12 \pm 0.05$ & 1.05 \\
\hline 200 & $1.22 \pm 0.05$ & 1.23 \\
\hline
\end{tabular}

$a_{\text {Average }}$ limb brightness over the height interval from $-3000 \mathrm{~km}$ to $+350 \mathrm{~km}$. 


\section{REFERENCES}

Avrett, E. H. 1985, in Chromospheric Diagnostics and Modeling, ed.

B. W. Lites (Tuscon: NSO), in press.

Beckers, J. M. 1972, Ann. Rev. Astr. Ap., 10, 73.

Beckman, J. E., Lesurf, J. C. G., and Ross, J. 1975, Nature, 254, 38 .

Cameron, R. M., Bader, M., and Mobley, R. E. 1971, Appl. Optics, 10, 211.

Clark, T. A., and Boreiko, R. T. 1982, Solar Phys., 76, 117.

Hagan, J. P. 1957, in IAU Symposium 4, Radio Astronomy, ed. H. C. van de Hulst (Cambridge: Cambridge University Press), p. 263.

Hagan, J. P., Swanson, P. N., Haas, R. W., Wefer, F. L., and Vogt, R. W. 1971, Solar Phys,, 21, 286.

Hermans, L., Limb-Intensity Profiles of Solar Chromosphere, 1984, Master's thesis, University of Hawaii.

Landman, D. A. 1984, Ap. J., $284,833$.

Lindsey, C., Becklin, E. E., Jefferies, J. T., Orrall, F. Q., Werner, M. W., and Gatley, I. 1983, Ap. J. (Letters), 264, L25.

Linsky, J. 1973, Ap. J. Suppl., 25, 163 .

Vernazza, J. E., Averett, E. H., and Loeser, R. 1973, Ap. J., 184, 605 (VAL).

. 1981. Ap. J. Supp1., 45, 635 (VAL) . 


\section{FIGURE CAPTIONS}

Fig. 1.--Schematic diagram of two-beam chopping-scanning mode used for observing the solar limb crescent. The geometry of the scan configuration approaching second contact is shown in a. The line segment scanned by the two beams is centered at the point of second contact, marked by an $x$. The profile plotted in $b$ shows the $0.5 \mathrm{~Hz}$ triangular-wave scan pattern of the center point of the two-beam pair. Profile $c$ shows the resulting two-beam difference signal profile as the positive and negative beams pass through the solar crescent. (Reproduction from Lindsey et al. 1983.)

Fig. 2.--Two-beam difference time series of scans across the solar crescent during second contact. Profile a shows the telescope scan position, $x$. It spans a distance of $5^{\prime}$, with positive pointing outward from the $1 \mathrm{imb}$ along the scan trajectory. Profile b shows the telescope response as the solar crescent passes through the positive and negative beam each scan. The amplitude of the envelope diminishes as the solar limb is occulted. Note, however, the significant lunar residual persisting after the sun has disappeared. Profile $c$ shows the solar limb profile reconstructed from profile b.

Fig. 3.--Intensity profiles of the second-contact limb are plotted for $200,100,50$, and $30 \mu \mathrm{m}$ radiation (solid curves in a, b, c, and $d$, respectively). Profiles of the VAL limbs (dashed curves), computed by Hermans (1984) are also shown. Here height is referenced to the bottom of the VAL photosphere $(\tau(5000 \AA)=1)$. Vertical arrows denote the visible limb, which appears at $340 \mathrm{~km}$ in VAL. The 
brightness is normalized to unity at disk center (left ordinate scale). It is also matched to the brightness temperature predicted by VAL (right ordinate scale). Error bars for $h>-1000 \mathrm{~km}$ denote a statistical uncertainty of \pm 1 standard deviation in intensity for 1" resolution. Error bars for $\mathrm{h}<-1000 \mathrm{~km}$ denote the statistical uncertainty for 2 " resolution.

Fig. 4.--Intensity profiles of the third-contact limb are plotted for $200,100,50$, and $30 \mu \mathrm{m}$ radiation (solid curves in a, b, c, and $d$, respectively). Profiles of the VAL limbs are also shown (dashed curves). Vertical arrows denote the visible limb. Error bars denote a statistical uncertainty of one standard deviation in intensity for $1^{\prime \prime}$ resolution.

Fig. A1.--Conceptual diagram of scheme for expressing telescope response in terms of line integrals. 


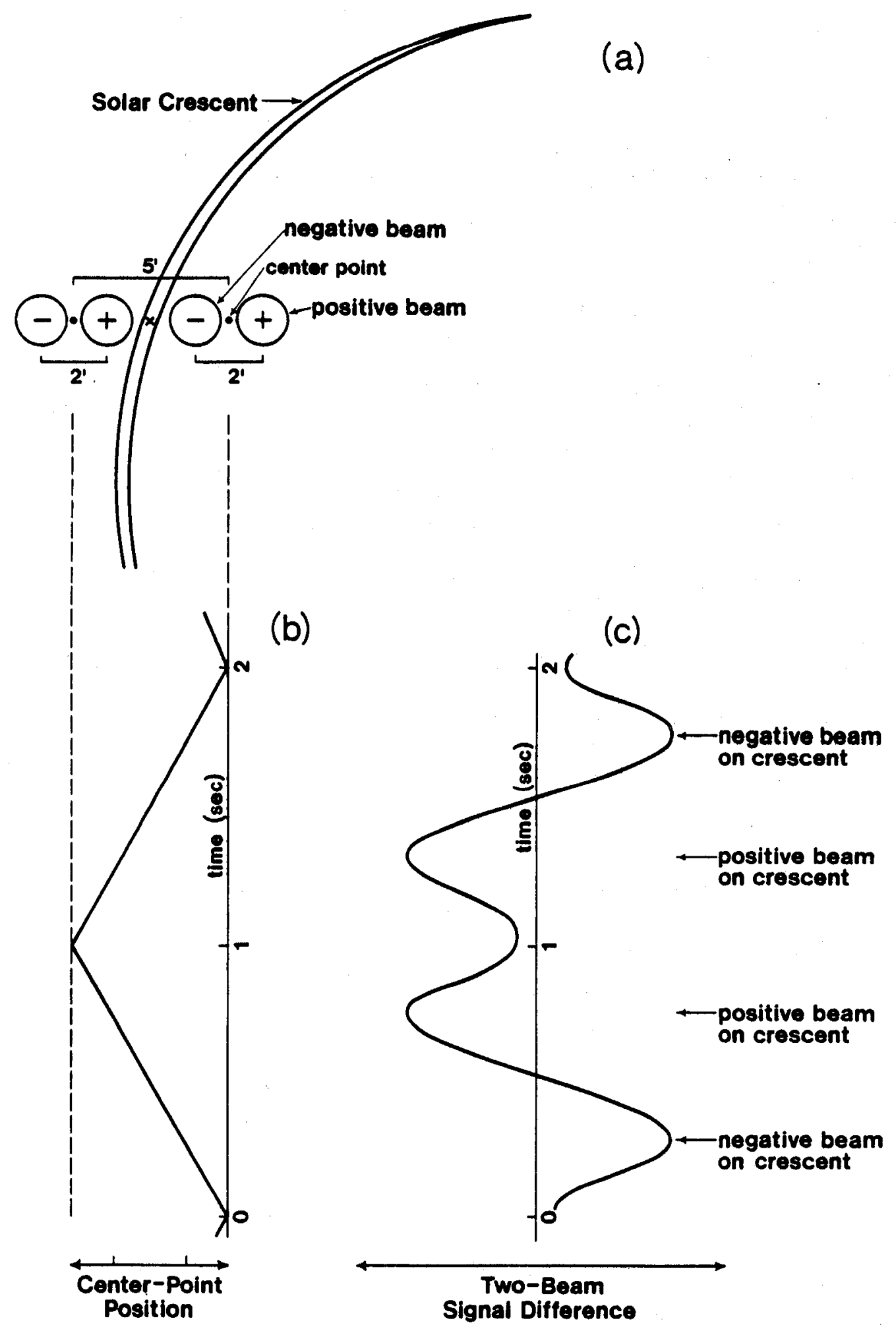

Fig. 1 


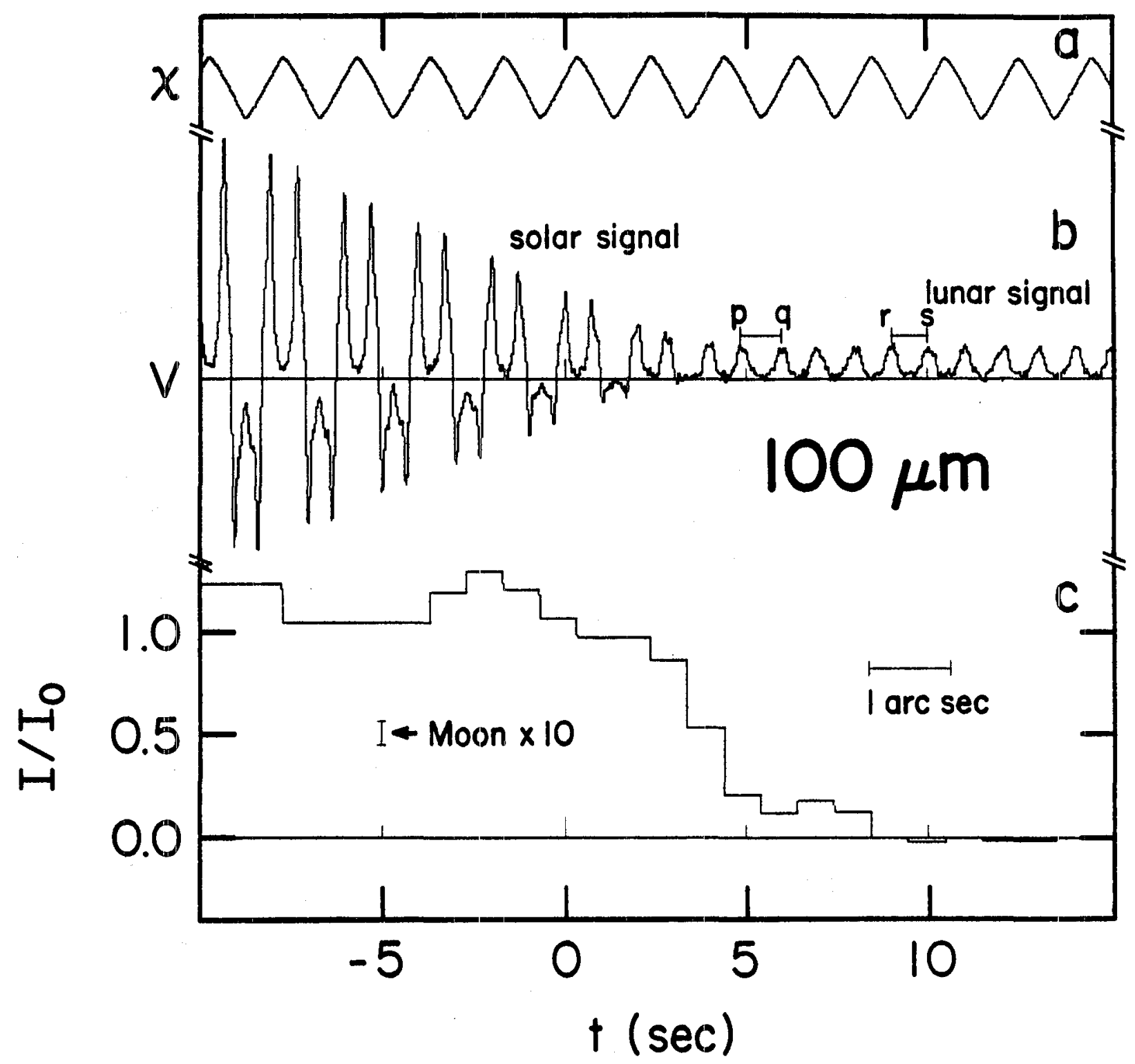

Fig. 2 


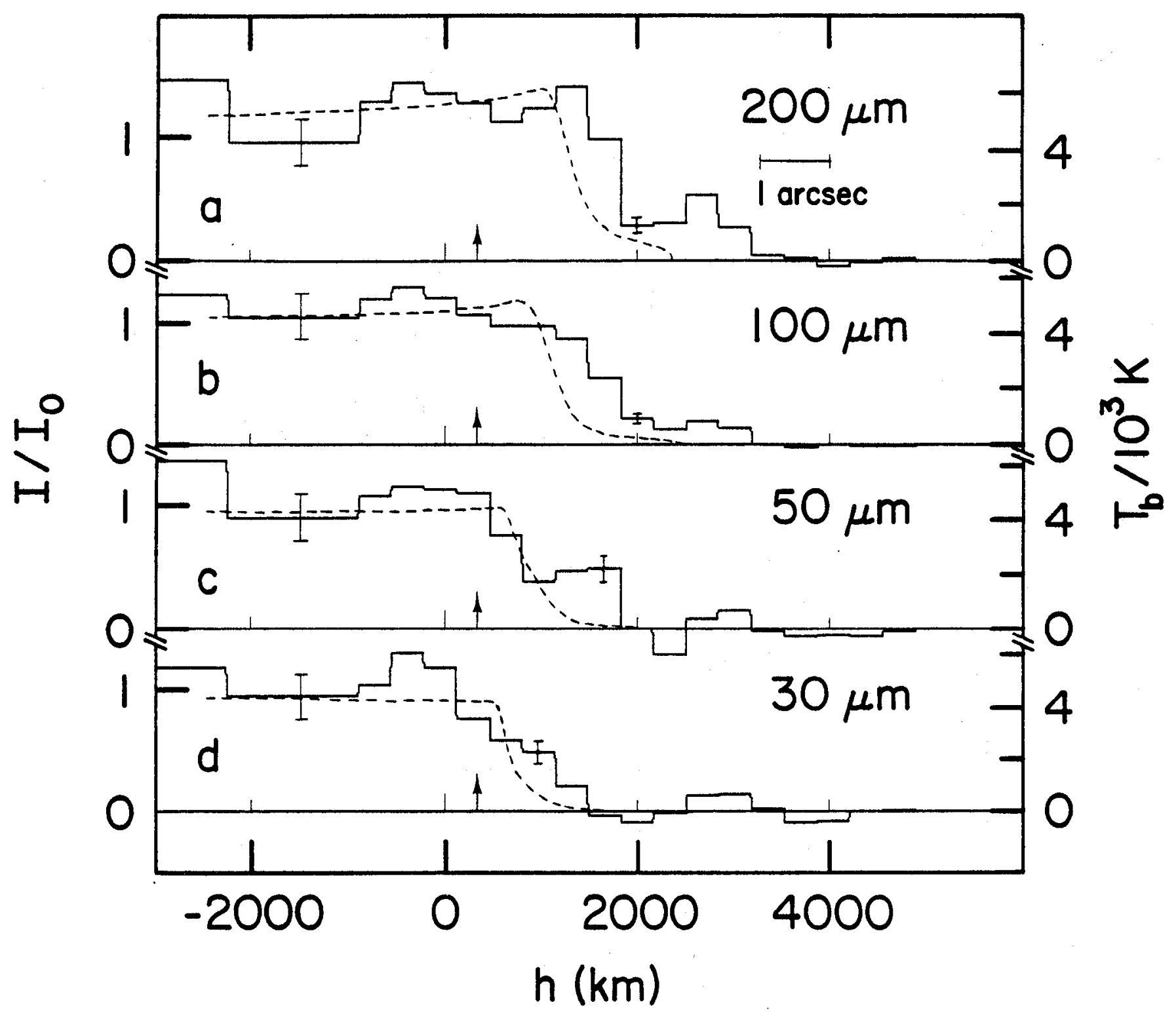

Fig. 3 


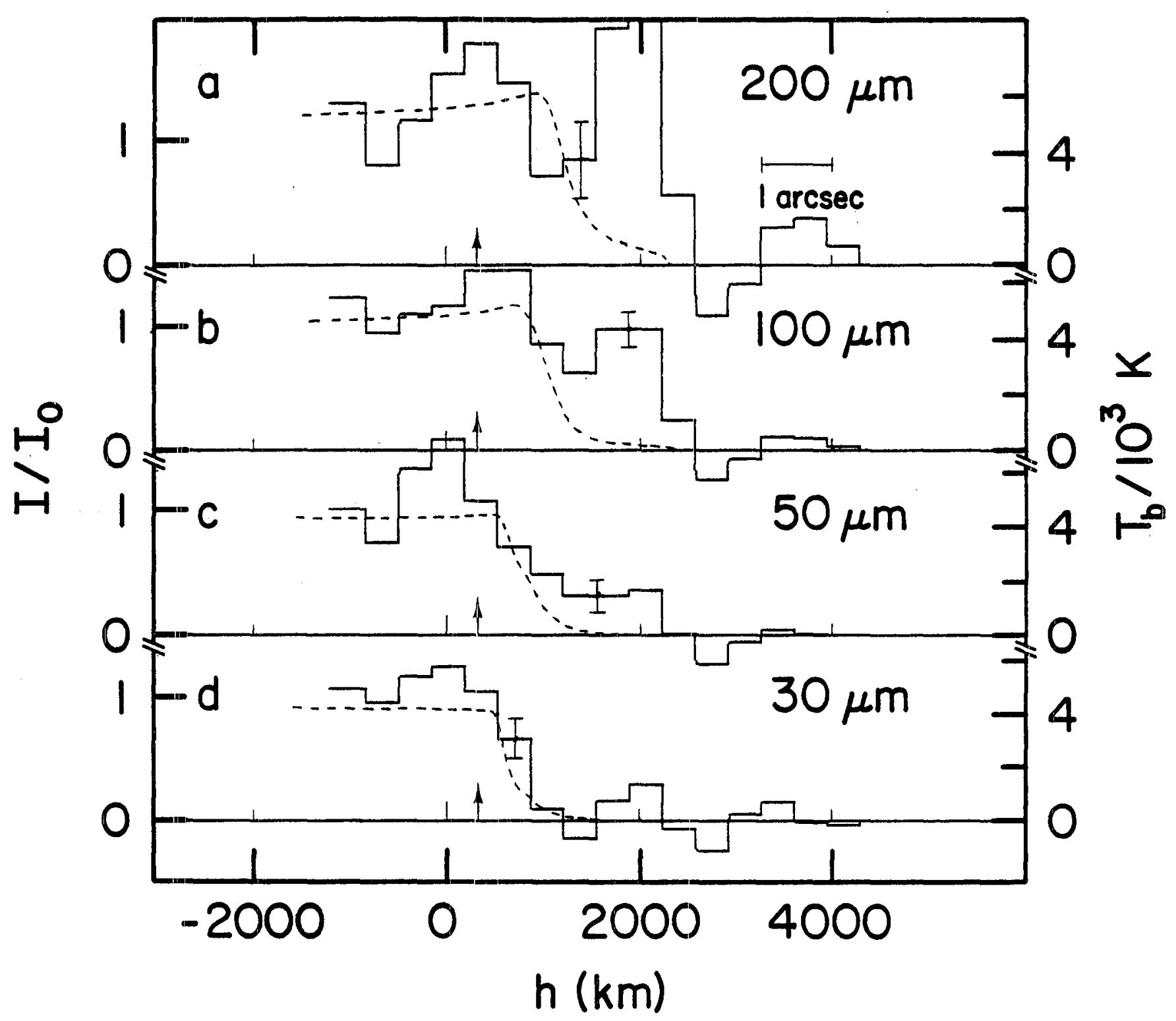

Fig. 4 


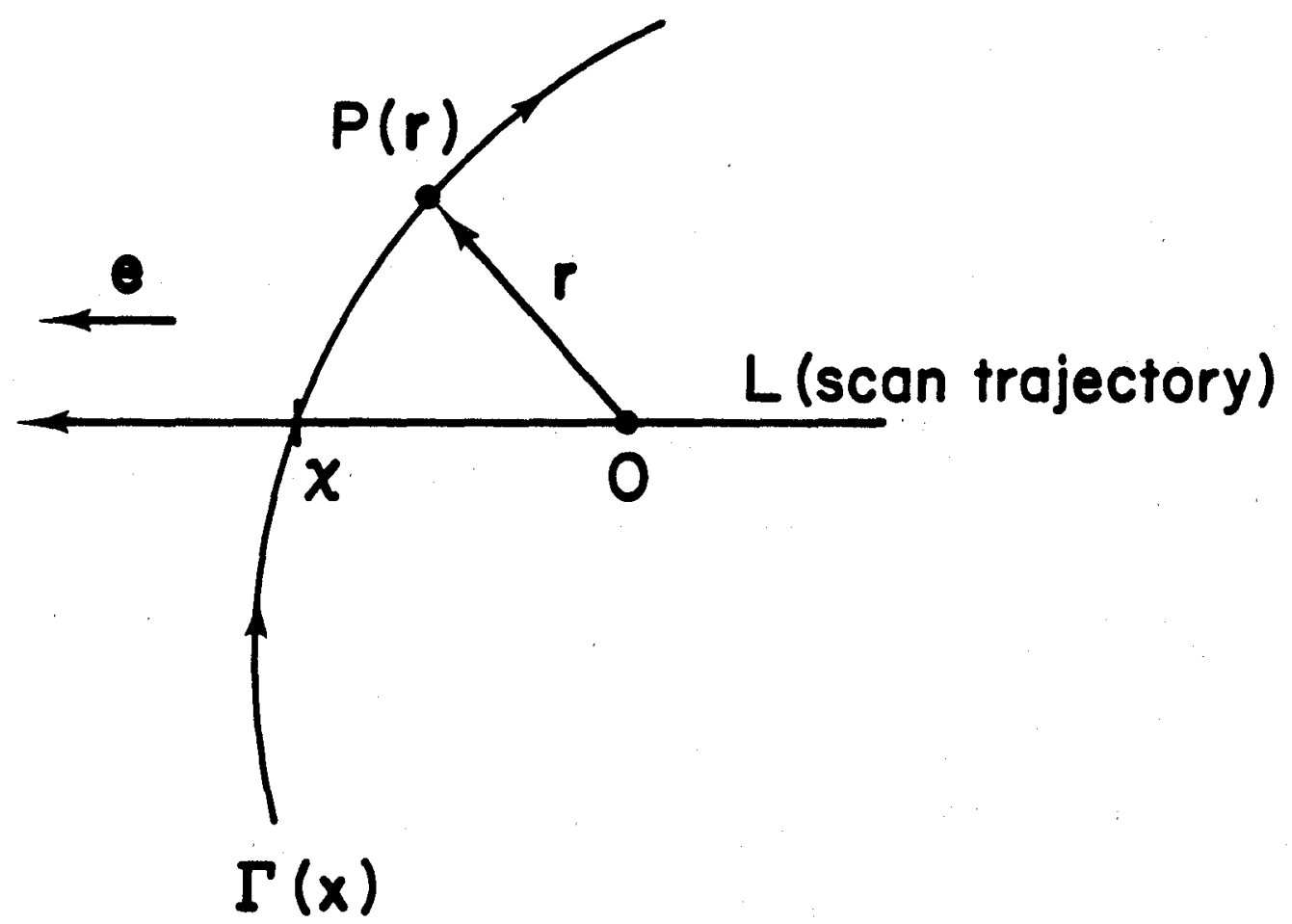

Fig. Al 


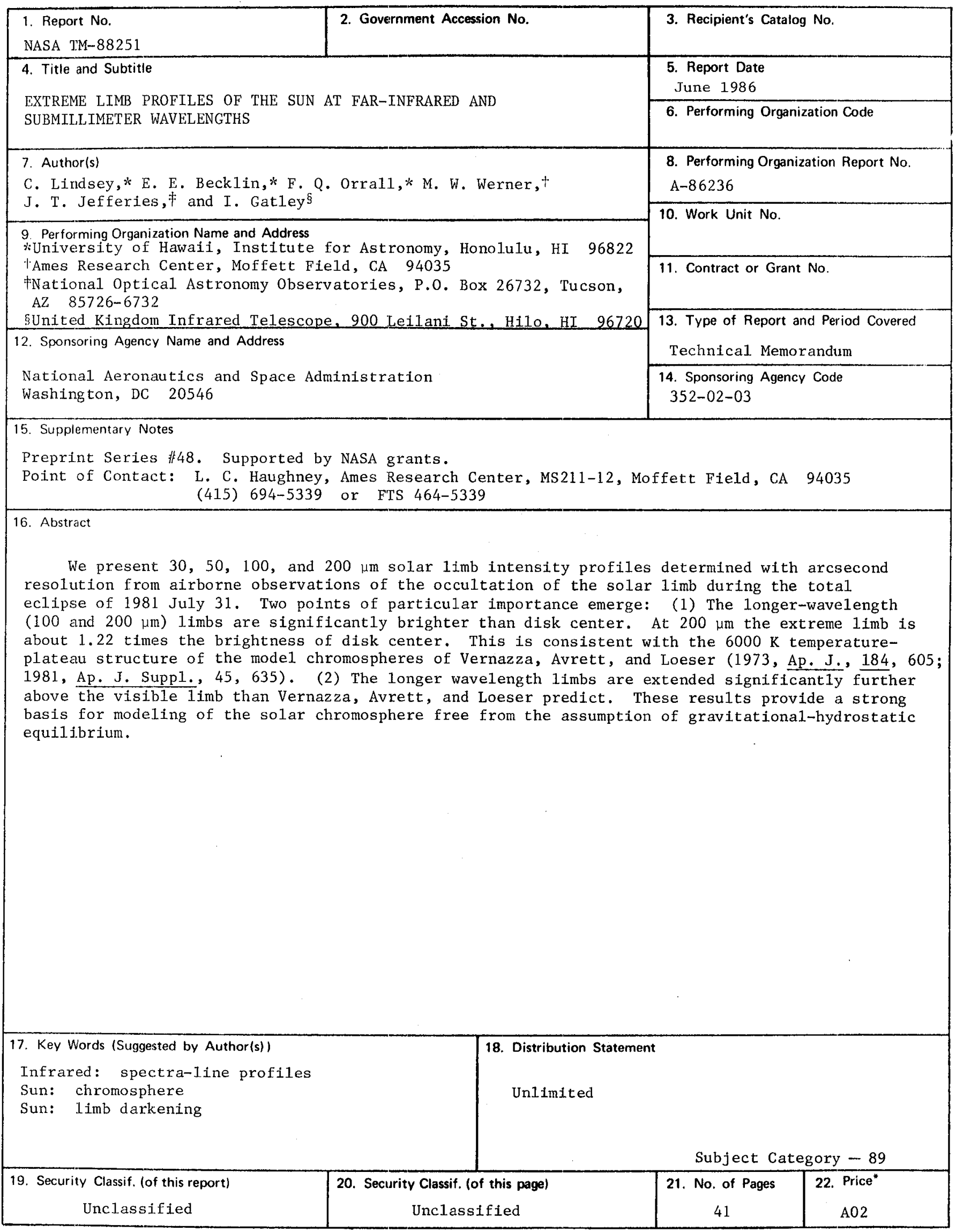

"For sale by the National Technical Information Service, Springfield, Virginia 22161 
End of Document 